\title{
Aortic surgery symposium 2010 discussions-Session V: Dissection and session VI: Descending/thoracoabdominal I
}

\author{
Moderator: Joseph E. Bavaria, MD, University of Pennsylvania Medical Center, Philadelphia, Pa. \\ Panelists: D. Craig Miller, MD, Stanford University School of Medicine, Stanford, Calif; Steven L. Lansman, \\ MD, PhD, Westchester Medical Center, Valhalla, NY; G. Michael Deeb, MD, University of Michigan, Ann \\ Arbor, Mich; Christoph A. Nienaber, MD, Universitatsklinikum Rostock, Rostock, Germany; Wilson Y. Szeto, \\ MD, Division of Cardiovascular Surgery, University of Pennsylvania, Philadelphia, Pa; Konstantinos Tsagakis, \\ MD, Department of Thoracic and Cardiovascular Surgery, West German Heart Center Essen, University Hospital \\ Essen, Essen, Germany; Marc Schepens, MD, AZ St. Jan, Brugge, Belgium; Randall B. Griepp, MD, Mount Sinai \\ School of Medicine, New York, NY; Anthony L. Estrera, MD, Cardiothoracic and Vascular Surgery, University \\ of Texas Medical School at Houston, Memorial Hermann Heart and Vascular Institute, Houston, Tex; Charles \\ Acher, MD, University of Wisconsin, Madison, Wis.
}

\section{Discussion}

Dr Bavaria. We will start some discussion from the floor.

Dr S. Vitale (Valhalla, $N Y$ ). I was just wondering whether you can elaborate on the use of naloxone for spinal cord protection because I have not seen any good data that support it.

Dr Acher. Yes, I can. There are no level I data, but the experimental data are quite clear that endorphins have a very negative effect on injured spinal neurons and that endorphin receptor antagonists are effective in protecting the spinal cord experimentally. It has also been shown lately that naloxone reduces the release of excitatory amino acids in spinal fluid of patients undergoing elective aortic resection-thoracoabdominal repair. This is by the Japanese author Kunihara in the Journal of Vascular Surgery in 2004. But more importantly, the issue for us when we introduced its use was the experimental literature on use of high-dose naloxone, which was unacceptable because of its analgesic reversal. Therefore the first patient we used it on was a patient with spinal fluid drainage who woke up with weakness, and I observed her getting progressively weaker over the next few hours. I called my anesthesiologist and said, "What is the lowest dose of naloxone that has a central nervous system effect?'; it was the dose that reverses apnea associated with epidural narcotics. We used that dose, and 2 hours later, the patient had normal neurologic function. We repeated that observation in the next patient who woke up weak with spinal fluid drainage and had the same result over a longer period of time and have used it ever since. We do think it is an important part of our protocol. We presented that in 1989. It was published in 1990 in our first series of 42 patients.

Presented at Aortic Surgery Symposium 2010, New York, NY, Friday, April 30, 2010. Disclosures: Joseph E. Bavaria is a member of the speakers bureau for Vascutek Terumo, and receives grant/research support from Gore \& Associates, Inc, and Medtronic, Inc, Vascular Therapy Division. Christoph A. Nienaber receives grant/research support from Medtronic, Inc; and is a member of the speakers bureau for Boston Science, Inc, and Cook, Inc. D. Craig Miller, Steven L. Lansman, G. Michael Deeb, Wilson Y. Szeto, Konstantinos Tsagakis, Marc Schepens, Randall B. Griepp, Anthony L. Estrera, and Charles Acher have nothing to disclose with regard to commercial support. Address for reprints: Eva B. Griepp, MD, Department of Cardiothoracic Surgery, Mount Sinai School of Medicine, New York, NY 10029 (E-mail: ebgriepp@aol. com).

J Thorac Cardiovasc Surg 2010;140:S142-6

$0022-5223 / \$ 36.00$

Copyright $\odot 2010$ by The American Association for Thoracic Surgery doi:10.1016/j.jtcvs.2010.07.049
Dr Bavaria. Marc, do you ever use any naloxone?

Dr Schepens. I have no experience with the use of naloxone.

Dr Bavaria. Drs Griepp and Estrera, do you use naloxone, except for rescue maybe?

Dr Estrera. No.

Dr Griepp. No, we have not, either.

Dr R. Calhoun (Sacramento, Calif). This is a more appropriately timed question today than yesterday, but it is the same question. For the occasional acute type A dissection in the operating room, in which it is clear that the tear is beyond the ascending aorta, either up in the arch or perhaps even more distally, I am curious about the philosophy of the panel in terms of your aggressiveness about going after that tear as opposed to just replacing the ascending aorta and trying to prevent the proximal malfeasance associated with tamponade. Correlatively, there is the occasional stent or some other process that has caused retrograde dissection into the ascending aorta, and it is clear that it is not a primary ascending aorta problem. How do you approach that?

Dr Bavaria. To clarify, what does the ascending aorta look like? Is it an intramural hematoma (IMH) of the ascending aorta, or is it a true full-blown double-barrel aorta?

Dr Calhoun. The ascending aorta has hematoma, but on inspection, the tear is perhaps up in the arch at the level of the carotid artery or somewhere where acutely going after it would require a much more extensive operation.

Dr Bavaria. Dr Miller, you have an acute type A aortic dissection with 2 barrels, and you have your primary tear site in the distal arch or descending aorta. What is your protocol?

Dr Miller. Or an acute type A dissection where you find a tear in the distal arch, I think was the question. I would go after it in a younger healthier patient. Why be afraid of going after it and doing the right operation? If it is in the proximal descending artery, however, which happens, then it is a lot stickier wicket. There is one neat trick we have not heard discussed yesterday or today, which is the Djumbodis device in Europe, which is a big bunch of nitinol that you throw down there, and it just gently pushes the flap out. That might help. Or you can do the open E-vita, which we have heard about from Essen, or you can do an antegrade stent graft. If it is way beyond and you are not going to be able to get to it directly surgically, you might want to think about that. I caution everyone when I say that, because Kazui himself has never been able to prove that the late reoperation 
rate was any lower in his young patients with Marfan syndrome, in whom he performed a total arch and a short elephant trunk, compared with his historical series. Therefore going the extra mile, doing something downstream, still lacks proof of efficacy, and we all know it has risk.

Dr Bavaria. Dr Deeb, you have a type A aortic dissection, and you get in there and really the primary tear site is in the distal arch or descending aorta. What do you do at Michigan?

Dr Deeb. I think our approach is similar to Dr Miller's. If it is in the arch and we can see it, we are very aggressive. If it is just beyond the left subclavian and I can get to it, I am very aggressive. If you can see it and it is beyond the left subclavian, we have done a couple of cases in which we have done hybrid procedures. We have stuck a stent down antegrade, put some mattress stitches in to secure it, and then we have sewn our distal to that and completed the arch. We will be very aggressive at getting the tear.

Dr Bavaria. That might be the best way to do it. Dr Lansman, do you have a comment?

Dr Lansman. I just wanted to add that over the years, I think we very rarely replaced the whole arch. We have a fairly large experience with acute dissections. In almost every case we can do a hemiarch with the caveat that some of those are very extended hemiarches, even extending into the proximal descending aorta. Fortunately, most of the tears are on the lesser curvature, but some extend toward the greater curvature, and most often you can fashion the hemiarch to eliminate the tear.

Dr Bavaria. Let's take that question a little further. You have an acute type A dissection by definition, I suppose, but the ascending arch component is really an IMH. The adventitial diameter is less than $5 \mathrm{~cm}$, the acute hematoma is less than $1 \mathrm{~cm}$, and there is no aortic insufficiency and no significant pericardial effusion. Would the panel treat this medically or with surgical intervention?

Dr Lansman. Well, I can start by saying we have an $\mathrm{N}$ of 1 , and it was an 87-year-old man with a type A dissection, just as you described, an IMH that presented after a couple of days, and we were happy to watch it. On a computed tomographic (CT) scan about a week or so later, it had converted to a real type A dissection, and the patient underwent surgical intervention successfully. After my review, we have decided to adopt the type of approach that Dr Estrera so nicely laid out.

Dr Bavaria. Therefore again, we will put it that you really have a type B dissection with retrograde IMH only, with the parameters I just described. Let's go down the panel. Dr Szeto, what would you do for that, medically or surgically manage or maybe a stent graft?

Dr Szeto. Well, Joe, as you know, we have struggled with that at our institution as well. A lot of it obviously will have to be guided by the patient's scenario. We tend to be more aggressive in younger patients, and in 80-year-old or older patients we tend to become more conservative. As you know, we have also had a recent experience with an 85-year-old woman with a similar clinical scenario as you described. We watched her, and she ruptured in the hospital. You remember that. Therefore it is a difficult question, but I think most of it, at least in our hands, is driven by clinical presentation and the status of the patient, and we tend to be more aggressive when they are younger.

Dr Estrera. Yes, I agree regarding IMH, and we published that. We are pretty aggressive. Having said that, you need to individualize, and obviously an older patient like that we might watch. But in the majority of those cases, we observe that most of them will convert, at least in the Western patient population down in Houston. We know that a lot of them convert, and therefore we are pretty aggressive.

I want to make one comment about the typical dissection that extends into the arch or where the tear is in the arch. Our philosophy has been to be less aggressive and just take care of the ascending and proximal arch. In our series of 366 patients, we did extensive arch procedures in fewer than $5 \%$ of the patients. And the reality of what we are seeing in follow-up, although I need to analyze these data, is that the arch, for some reason, does not dilate that much. It is really the proximal descending aorta that dilates. If the tear starts at the proximal descending aorta, I will not go after it. I will take care of the problem that is going to kill the patient, which is pericardial tamponade. I will take care of the ascending aorta first.

Dr Bavaria. Christoph, is there any role for stent grafting in the scenario in which you have a type B dissection with a retrograde proximal IMH?

Dr Nienaber. Well, if you are talking about IMH, you have to consider that no tear is available. Therefore there is actually by definition no type B dissection with an IMH. It is extending to the proximal aorta but is basically a dissection in evolution, and that is considered to be an indication for surgical intervention as long as, in type B dissection, the proximal aorta is involved. Therefore I would not recommend any stenting in a situation like that, which is a growing involvement of the ascending aorta. An IMH in the ascending aorta has to be individualized. We consider it a dissection in evolution and only very sick comorbid patients are patients to be watched; others should undergo operations in an elective fashion very soon.

Dr Griepp. I just had a question for the other panelists. I wonder whether anybody had ever seen cerebral embolization in a type A IMH that converted to a type A dissection. Where did all the clot go?

Dr Bavaria. Good question. I have seen cerebral malperfusion from a true lumen collapse. I do not think I have ever seen embolization. Has anybody on the panel ever seen that?

Dr Acher. Actually, we did have a patient with Marfan syndrome with an acute type $\mathrm{B}$ dissection in whom we had done a type IV, quite large aneurysm a few years before. One of the complications of his type B dissection was a stroke, not from malperfusion but from an embolus because of the proximity of the dissection to the left carotid artery.

Dr G. Di Luozzo (New York, NY). This question is actually for Wilson. In your series of complicated type B dissections treated with endovascular techniques, what was your paraplegia rate? After paving a descending thoracic aorta extensively, how do you manage the blood pressure (because that could be tricky)? Do you put lumbar drains in these patients?

Dr Szeto. Those are very good questions. In our series, strikingly and contrary to the other series published, the risk of spinal cord ischemia was not insignificant. Our rate of spinal cord ischemia was almost $13 \%$. Having said that, most of these were not permanent: only 2 of these patients actually had permanent spinal cord ischemia resulting in paraplegia.

In terms of how we would manage these patients intraoperatively, at least in this population, the acute complicated patients 
who present in extremis, in general we tend not to place a lumbar drain preoperatively because of the emergent nature of the operation. If neuromonitoring is available, we use somatosensory evoked potentials and motor evoked potentials (MEPs), and postoperatively we tend to try to wake the patients earlier to get a more reliable neurologic examination. If clinically necessary, we intervene with either a lumbar drain or volume expansion and so on. Therefore it is a difficult situation or scenario. These patients are often very sick.

Blood pressure management, I agree, is quite tricky. In patients with malperfusion, we tend to be a little bit more liberal with having a higher blood pressure as opposed to patients with rupture, but it is a fine balance.

Dr Griepp. I just wanted to ask whether any of those patients have had compromise of the hypogastric circulation caused by their dissection. Because the data suggest that if you have had a previous abdominal aortic operation and then you have a thoracic aortic operation, your incidence of paraplegia is higher. I suspect that many of those cases of spinal cord injury are explained by loss of the hypogastric circulation at the abdominal operation.

Dr Szeto. You are absolutely right. One of our patients had previous abdominal aortic aneurysm surgery. However, what was common for both of those patients was extensive coverage of the thoracic aorta. One of them was a man with previous abdominal aortic surgery who had a rupture that we covered from the left subclavian artery down to the celiac artery. The second patient had severe malperfusion that required a so-called full metal jacket, where just 1 endograft proximally covering the primary tear site was not enough to reverse the malperfusion syndrome, and he had subsequent distal endografts. There were not only covered stents in the thoracic aorta but also bare metal stents in the infrarenal abdominal aorta and the iliacs. Therefore even though the numbers are small and I cannot make strong conclusions based on that, I agree that hypogastric compromise is certainly a risk factor for which to watch.

Dr B. Youdelman (Philadelphia, $P a$ ). We have had 2 patients in whom we were doing stent grafts for acute type B dissections who had retrograde hematomas. One progressed as we were following him, and we wound up doing a type A repair. The other we watched. I have not heard the incidence of the retrograde hematoma or type A dissection from the group, and I was wondering what everybody is seeing with their stent grafts for acute dissection and what everybody is doing about them.

Dr Miller. Everybody has his or her numbers, and the incidence is very high. It is probably a function of the type of stent graft you are using for the acute complicated type B dissection. The Talent and maybe even the Valiant grafts are the worst offenders based on the European cooperative study and the data from China and Shanghai; the number of stent grafts being used in China is phenomenal. However, they have a serious retro A problem, and the stent graft used in Shanghai, I think, is a knockoff of the Talent. There was also a recent report from Italy with a very high incidence. As we all know, these retrograde hematomas can be highly lethal. It depends on the type of stent graft. I do not think it depends on how much you cover.

Dr Bavaria. Christoph, you have personal experience with this. What is your personal retrograde type A dissection rate for all the stent grafts that you put in for dissections?
Dr Nienaber. Fortunately, it is low. But in the Investigation of Stent Grafts in Patients With Type B Aortic Dissection (INSTEAD) study we mentioned, we have one case that was probably due to a pre-existing IMH that was not picked up on initial imaging. However, returning to the reason for retrograde dissection, I think it is not only the type of stent graft but also the handling. There should be no poststent ballooning in the proximal end of the stent, there should be no oversizing, and there should be, obviously, no aggressive proximal end of the stent graft that could potentially cause rupture or penetration through the intimal layer of the affected aorta. Therefore there are various elements. One is the nature of the stent, but more important, I personally think, is the handling of the procedure.

Dr Bavaria. Wilson, in the 45 patients that you had, what was your retrograde type A dissection rate?

Dr Szeto. As you know, we had 3.

Dr Bavaria. That is $6 \%$.

Dr Szeto. We had 3, and they were delayed. They were all found on a postoperative surveillance scan at 3 months or later.

Dr Bavaria. To echo Dr Miller, I have just reviewed 500 of these from Hong Kong. The retrograde dissection rate was 3\% for acute dissections, both complicated and uncomplicated batched together.

Dr Miller. Before we leave that topic, I think it behooves the manufacturers to come up with a dissection-specific device: maybe the LP Zenith by Cook is going to be that, with nitinol instead of stainless steel, or maybe the Gore C-TAG might be a little kinder and gentler on the proximal arch.

Dr Bavaria. We probably are going to get better stent grafts. That is going to have to be key, or else we are not going to be able to go much farther with uncomplicated type B dissection cases. That is for sure.

Dr D. Spielvogel (Valhalla, $N Y$ ). This is for the panelists but also for Dr Deeb. I have been intrigued by your approach to the difficult patients with malperfusion. We approach malperfusion with fenestration as you illustrated, and we do not use any endografts. The endografts with fenestration you do not see. We get retrograde type A dissections, and we do not see paraplegia. My question really is this: Have you looked at the group of patients with rupture, and is there a way to tease out that group and maybe intervene before rupture? When we talk about malperfusion, we kind of lump it all together. What we do not see are data like the $\mathrm{pH}$ of the patient and the level of lactic acidosis. The patients who do very poorly are the acidotic patients with visceral malperfusion: superior mesenteric artery occlusion or obstruction. Therefore is there a way of separating out that group in whom one can intervene and prevent loss through rupture?

Dr Bavaria. So, Mike, answer that. But also, you had a lot of ruptures. How many of the ruptures were from type A ascending stuff, and how many were actual ruptures from the fenestration procedure distally?

Dr Deeb. They were all ruptures in the proximal descending thoracic aorta. We autopsied them. None of them were retrograde type A: we didn't have those. None of them were down in the abdomen where we had done the fenestration. They were all in the proximal descending thoracic aorta. You can say that the procedure did not have anything to do with it. My answer is that it did because we changed the hemodynamics. We went in and instrumented the patient. Doing that procedure does not decrease the risk of rupture 
that you have from these patients. Obviously in those patients who have no complications and yet are treated medically, the big risk is rupture. The incidence of that is relatively low. This incidence was $7 \%$, I believe. I think it is slightly higher than in those treated medically, and it might have something to do with the instrumentation that we did on these patients. We have not looked at these patients specifically and run risk factors on them. That might be something that we should do, to try to identify who is at risk of rupturing. As for this patient population, once identified, should they have an endovascular approach rather than the percutaneous fenestrationstent? Are these patients you would go right after with thoracic endovascular aortic repair (TEVAR)?

Dr Miller. Well, both of you are taking the fenestration-stent experience as the benchmark to which we should compare TEVAR for acute complicated B.

Dr Deeb. I am glad you asked that. No, we really were not.

Dr Miller. I am glad you are not because you are going to lose.

Dr Deeb. What we did is that we read the literature, and everyone said that we have to get better technical devices to work with, that we need to gain more data, and that we need to compare TEVAR with another procedure. When we put in our article for the American Association for Thoracic Surgery and we read the conclusion, we said that fenestration-stent should be used for comparison. I looked at my young partner, Himanshu, and said that this approach was going to get trashed. Therefore what we need to do is put something out there that is going to elicit a reaction. I said that we should put a benchmark out there, and it seemed to work because we got it on the program. We do not think the fenestration-stent is a gold standard to which one should compare anything, but we do know it is a procedure that has been out there that has some effectiveness, and we can get some datadriven outcomes. Everyone wants data-driven outcomes. Here is something we have done for a long time.

Dr Miller. But there are data now, and, David, what you are doing now makes me really surprised. Why? It is a benchmark, and it is excellent, but if you look at that late attrition, you do not see that in the Penn group or in our 16 patients. You do not see that late attrition if you cover the primary intimal tear with a short stent graft.

Dr Bavaria. Wait a minute. We have 1000 clinical aortic surgeons out here. I want to go right down this panel. You have an acute complicated type B dissection. Is the first order of therapy a stent graft, or is fenestration the first order of therapy?

Dr Deeb. We did fenestration and stenting because we had nothing else. There is no approved stent graft in the United States to use for type B dissection. Our institution highly discourages us from using it off label. Now that we are in the C-TAG trial, we are going to stent endovascularly. We are going to get some experience with that. If we do not see a marked difference, we are thinking about doing a randomized prospective trial.

Dr Schepens. Stent grafting.

Dr Acher. We also are in the C-TAG trial, and therefore we would only stent those. We published a series of medical management of about 81 type B dissections, $20 \%$ of which were complicated.

Dr Bavaria. The medical management of complicated type B dissection has a mortality of between $30 \%$ and $70 \%$.

Dr Acher. That is what we found when we operated.
Dr Nienaber. There is clearly no room for primary fenestration. The first step would always be stent grafting in cases of malperfusion.

Dr Bavaria. I think we are getting a consensus. Anybody else? We know Dr Miller's results.

Dr Estrera. You have got to cover the tear with a stent graft.

Dr S. Takamoto (Tokyo, Japan). Dr Bavaria told us about IMH and type $\mathrm{B}$ dissection. What he described is thrombus formation in the false lumen in the ascending aorta: that is a complete misconception of IMH. IMH should not have a tear. For the study in Japan of about 200 type B dissection-related IMHs, that was the initial diagnosis. In one third of the patients, a false lumen becomes patent. Therefore sometimes the tear is hidden at the time of initial diagnosis. Only the CT examination shows that diagnosis. However, if I see images of a false lumen obtained by means of transesophageal echocardiographic analysis, the false lumen is not completely thrombosed at the first moment: in some parts the thrombus is there, but in other parts fluid exits and is moving. IMH is not an accurate diagnosis for surgeons; it is terminology for radiologists and is not good for surgeons. We should take care of that kind of disease as aortic dissection with a thrombosed false lumen. What do you think about that? IMH is not an appropriate term for surgeons.

Dr Bavaria. My point is that you have this proximal aortic event, which is kind of an IMH, but the primary problem is in the proximal descending aorta, and how should you approach that? The problem is that if we do a sternotomy and an ascending hemiarch and whatever else, this is not really affecting the major issue. We might be doing kind of a lesser operation conceptually than perhaps we should be doing, which is to tackle the primary event. Maybe just coming across the primary tear site with a stent graft, for example, might depressurize the whole thing and stop the process from going any further. It was the concept that we, as cardiac surgeons, are going in through the front when the major problem is coming in from the back.

Dr Takamoto. What you are describing is an acute aortic dissection with a thrombosed false lumen in the ascending aorta. That is not an IMH. An IMH should not have a tear.

Dr Estrera. Dr Takamoto is exactly correct. IMH really is a radiological diagnosis, if you think about it. We just cannot see that tear by means of CT scan, and that was one of the flaws of our article. We made that diagnosis based on a CT scan or on intraoperative findings. The reality is that there are probably a lot of those that occurred between our screening in terms of identifying $\mathrm{IMH}$, and there probably is a tear that you just cannot see on a CT scan.

Dr Bavaria. I agree with that. I want to do a couple of questions, but there is one question I want to ask Drs Griepp and Estrera. I have heard Dr Griepp say that he has never seen any MEPs decrease after thoracoabdominal surgery, and Dr Estrera says it is $50 \%$ or greater. Can you guys educate us about the MEPs?

Dr Griepp. I suspect Dr Estrera has much more rigid criteria than we do. What I have said and I think is true is that we almost never see a situation in which the MEP decreases that we cannot get it back to where it was a few minutes before with hemodynamic manipulation. I am sure it has occurred once.

Dr Estrera. We do have pretty strict criteria that are used by a neurophysiologist at the time. You have got to think about the 
difference in our techniques. Dr Griepp's technique is different in that he will expose everything and then clip the intercostals off consecutively in a pulsatile patient. Our technique is that we clamp the aorta, fillet it open, and then we see whether neuromonitoring changes occur. Therefore it is really a different physiology. We have no pulsatile flow; Dr Griepp has pulsatile flow: this is a significant difference between approaches. I think what needs to be recognized is the concept of a collateral network that he has established because I think this changes the way one thinks about how to protect the spinal cord.

Dr S. Yetman (New Haven, Conn). I have a question for the panel in terms of your management strategy in the patient who presents with acute aortic dissection and peripheral neurologic deficits. The patient is still interactive, and therefore we are not talking about coma or anything like that. What are your parameters in taking this patient to the operating room? Is there a time interval from the onset of symptoms, and if there is a time interval, what medical management would you provide for this patient? Does it include the use of heparin?

Dr Bavaria. Michael Deeb, do you want to start off with that one as a kind of a delay guy?

Dr Deeb. That is an interesting question because we just had a young male patient who presented with a dense hemiplegia who was awake, and the question was, should we take him to the operating room? As we were moving this patient from the emergency department to the intensive care unit, he became somnolent. We got a CT scan of the head; it showed a small infarct in that area, and I thought that if I heparinized the patient and froze him down, he would not be getting any better. Some people were pushing me to do it. We sent him for a magnetic resonance angiography: he had a big area of infarct, and within 2 hours, he herniated and died. Therefore that is a very tough question. I do not think one does anyone who has a significant central neurology intracranial deficit any good by cooling down their brain and heparinizing them.

Dr Bavaria. Dr Tsagakis, what are you doing at Essen?

Dr Tsagakis. With a central neurologic deficit?

Dr Bavaria. Yes.

Dr Tsagakis. It depends, of course, on the condition of the patient. If the patient is awake, then we will operate: this is clear. If the patient is not and we have imaging of the brain, which is not very common, on which we see a very big injury on the left side of the brain, we do not perform the operation. We had such a case last year.

Dr Bavaria. Your follow-up question was about timing?

Dr Yetman. Yes. Is there any timing involved in terms of the onset of symptoms versus when they present to you that determines whether you operate on the patient?

Dr Tsagakis. With regard to neurologic injury, actually you have no time. You must operate or not.

Dr Bavaria. I think that is an important question. If the neurologic symptoms have been there for a while, we would usually not take them to the operating room right away if there is a big, dense neurologic deficit. However, if the patient presents in less than 6 to 8 hours, we would actually take those patients to the operating room and reperfuse them, and we have had fairly reasonable results with that. It is not $100 \%$, obviously, but it is fairly reasonable.

Unfortunately, we are going to have to cut it off here. Thank you very much. 